\title{
Nature of the rabbit acrosome reaction-inducing activity of follicular fluid
}

\author{
Gene Oliphant, Christine L. Cabot and C. A. Singhas \\ University of Virginia, School of Medicine, Division of Reproductive Biology, \\ Department of Obstetrics and Gynecology, Charlottesville, Virginia 22903, U.S.A.
}

\begin{abstract}
Summary. Follicular fluid samples from rabbits, cats, pigs, women and cows had acrosome reaction-inducing activity (ARIA) on rabbit spermatozoa as determined by differential staining after incubation with these fluids. Activity was retained after dialysis and at least $50 \%$ was found to be labile when heated to $56^{\circ} \mathrm{C}$ for as little as $20 \mathrm{~min}$. The induction of the acrosome reaction by bovine follicular fluid showed a dependence on the concentration of follicular fluid and spermatozoa and on calcium ions, and had a pH optimum of approximately 8. Enzyme treatments showed that proteases destroyed the ARIA and this activity was completely blocked by treatment of bovine follicular fluid with goat anti-bovine antiserum. Electron microscope observations indicated the similarity of the reactions observed to that occurring in vivo. It is concluded that the rabbit acrosome reaction-inducing activity of bovine follicular fluid is a serum component(s), probably a protein(s) of high molecular weight.
\end{abstract}

\section{Introduction}

The sperm acrosome, thought to be a differentiated lysosome (Fawcett, 1958), apparently functions during the fertilization process by releasing its contents of hydrolytic enzymes and thus facilitating sperm penetration through investments which surround the ovum. The process by which the enzymes may be released is the result of fusion and vesiculation of the plasma membrane specifically covering the acrosome and the outer acrosomal membrane. This process has been termed the acrosome reaction and occurs universally in mammalian spermatozoa (Barros, Bedford, Franklin \& Austin, 1967). Ovarian follicular fluid has been shown to induce a functional acrosome reaction in hamster (Yanagimachi, 1969) and mouse (Iwamatsu \& Chang, 1969), and to facilitate fertilization of rabbit ova in vitro (Rosado, Hicks, Reyes \& Blanco, 1974; Oliphant, 1976).

The work presented in this paper attempts to provide data on the acrosomal changes induced in rabbit spermatozoa by follicular fluid, thus defining the rabbit system for further study and further characterizing the component(s) of follicular fluid affecting the sperm acrosome.

\section{Materials and Methods}

Follicular fluid was obtained from bovine or porcine ovaries collected from an abattoir. Ovaries were placed on ice immediately upon removal from the animal. Within $2 \frac{1}{2}$ to $3 \frac{1}{2} \mathrm{~h}$ fluid was aspirated from the follicles, pooled, divided into $0.5 \mathrm{ml}$ aliquots, and stored frozen at $-20^{\circ} \mathrm{C}$ until use. Human follicular fluid was collected by aspiration of follicles at the time of surgical procedures. Rabbit and cat follicular fluids were obtained from normal ovaries of animals used in this laboratory.

Rabbit spermatozoa were collected from New Zealand White bucks of proven fertility by use of an artificial vagina. The sperm concentration was determined with a haemocytometer and the ejaculates diluted to the required concentrations with CB medium (Cross \& Brinster, 1970). Spermatozoa exposed for $5 \mathrm{~min}$ to an environment of 380 mosmol by treatment with a modified Krebs-Ringerbicarbonate medium adjusted with $\mathrm{NaCl}$ : this was the High Ionic Strength (HIS) medium (Brackett \& Oliphant, 1975). A volume of $25 \mu \mathrm{l}$ of a suspension of pretreated rabbit spermatozoa was then transferred to the mixture for induction of the acrosome reaction. The reaction mixture, maintained at $37^{\circ} \mathrm{C}$ under paraffin oil, consisted of $50 \mu \mathrm{l} \mathrm{CB}$ medium equilibrated with $5 \% \mathrm{CO}_{2}$ in air and $50 \mu \mathrm{l}$ of an 
acrosome reaction-inducing agent. For control treatments, $50 \mu \mathrm{l}$ medium was used instead of inducing agent. These conditions were altered only during determination of the concentration dependence of the acrosome reaction-inducing activity in follicular fluid when appropriate volumes (to a total of $125 \mu \mathrm{l}$ ) of medium and follicular fluid were used to obtain the desired follicular fluid concentration. When necessary for $\mathrm{pH}$ dependence assays, the standard medium was altered by addition of $\mathrm{HCl}$ or $\mathrm{NaOH}$. Follicular fluid was dialysed against the adjusted medium for 12-18 h before use. The $\mathrm{pH}$ in the small volume of the reaction mixture was tested with litmus paper before and after the experiments to show that the buffering capacity was sufficient in each case to maintain the required $\mathrm{pH}$ during the reaction times.

For each assay, the time course of the reaction was followed by taking $25 \mu \mathrm{l}$ samples at 0,60 , and 120 min of incubation. The samples were fixed and stained by the method of Wells \& Awa (1970) and 50-100 spermatozoa were counted for each sample to evaluate the completion of the acrosome reaction. Only spermatozoa from which acrosomes were completely removed were considered as reacted. For electron microscopy, samples were fixed in $3 \%$ glutaraldehyde and post-fixed in osmium tetroxide. Sections were dehydrated in graded acetone, embedded in epon, sectioned and stained with uranyl acetate.

\section{Follicular fluid treatments}

Each of the enzymes (purest commercially available) used for treatment of follicular fluid was dissolved in CB medium at a concentration of $5 \mathrm{mg}$ protein $/ \mathrm{ml}$. Activities of these enzymes were: pronase, 45,000 PUK units/g; trypsin, $2 \times 10^{6} \mathrm{BAEE}$ units/g; $\alpha$-amylase, $5000 \mathrm{SKB}$ units/g; $\beta$-amylase, 785,000 maltose units/g; lysozyme, $175 \times 10^{5}$ units/g; lipase, 3000 Wilson units/g. Enzyme solutions were substituted for the CB medium to test the direct effects of these enzymes on spermatozoa. Follicular fluid was incubated with these solutions for $10 \mathrm{~min}$ before introduction of spermatozoa. Follicular fluid was also pretreated with trypsin which was bound to agarose(Miles Laboratory) to give 38.4 BAEE trypsin units/200 $\mu \mathrm{l}$. Preliminary experiments indicated that little if any trypsin was solubilized from the agarose gel. For equilibrium, the bound enzyme $(200 \mu \mathrm{l})$ was suspended in $0.5 \mathrm{ml}$ follicular fluid for $5 \mathrm{~min}$ and recovered by centrifugation $(10,000 \mathrm{~g}, 15 \mathrm{~min})$. The bound trypsin was then suspended in $0.5 \mathrm{ml}$ of the follicular fluid to be tested. After reaction for $10 \mathrm{~min}$ at $4^{\circ} \mathrm{C}$ and with continuous swirling the follicular fluid was separated from the bound enzyme by centrifugation $(10,000 \mathrm{~g}, 15 \mathrm{~min})$.

The effect of $\mathrm{Ca}^{++}$on the acrosome reaction was determined. Follicular fluid was dialysed against $10^{-3} \mathrm{M}$-EDTA, $\mathrm{pH} 7 \cdot 0$, with an effective dilution of $1: 2 \times 10^{4}$ and then half of the sample was dialysed (effective dilution $1: 2 \times 10^{4}$ ) against $\mathrm{CB}$ medium (normally containing $\mathrm{Ca}^{++}$) and the other half was dialysed against $\mathrm{CB}$ medium without $\mathrm{Ca}^{++}$. The spermatozoa in the assays were initially washed in phosphate-buffered saline to remove calcium from the ejaculate, and $\mathrm{Ca}^{++}$-free $\mathrm{CB}$ was used for both the HIS medium treatment and dilution of follicular fluid.

Attempts were also made to inhibit the acrosome reaction immunologically. Goat anti-bovine antiserum (Cappel Labs) was heated at $56^{\circ} \mathrm{C}$ for $30 \mathrm{~min}$ and dialysed against $\mathrm{CB}$ medium (without BSA). This antiserum preparation $(50 \mu \mathrm{l})$ was used instead of the $\mathrm{CB}$ medium in the assay mixture. Antiserum was allowed to react with follicular fluid for $30 \mathrm{~min}$ at $37^{\circ} \mathrm{C}$ before introduction of spermatozoa.

\section{Results and Discussion}

\section{Species variation, dialysis and heating}

The acrosome reaction of rabbit spermatozoa preincubated in HIS medium was readily induced in human, cow or pig follicular fluid, and less so in cat and rabbit follicular fluids (Table 1). Only 2-3\% of spermatozoa incubated in control CB medium lost their acrosomal caps, presumably through autolysis. These results are consistent with previous reports that bovine follicular fluid induces the acrosome reaction in hamster (Yanagimachi, 1969) and mouse (Iwamatsu \& Chang, 1969) and that human follicular fluid induces the acrosome reaction of mouse, rat and human spermatozoa 
Table 1. Induction of the rabbit acrosome reaction ( $\%$ completion) by follicular fluids of various species

\begin{tabular}{lccc}
\hline \multicolumn{1}{c}{ Species } & Untreated & Dialysed & $\begin{array}{c}\text { Heated } 30 \mathrm{~min} \\
\text { at } 56^{\circ} \mathrm{C}\end{array}$ \\
\hline Man & $38 \pm 7 \cdot 4$ & $30 \pm 7 \cdot 5$ & $15 \pm 1 \cdot 0^{*}$ \\
Cow & $32 \pm 9 \cdot 9$ & $33 \pm 8$ & $16 \pm 2 \cdot 6^{*}$ \\
Pig & $30 \pm 3 \cdot 3$ & $34 \pm 7$ & $16 \pm 3 \cdot 1^{*}$ \\
Cat & $17 \pm 4 \cdot 2$ & - & - \\
Rabbit & $12 \pm 4 \cdot 6$ & - & - \\
\hline
\end{tabular}

Values are mean \pm S.D. for 6 determinations. The $1 \times 10^{7}$ spermatozoa/ $\mathrm{ml}$ were incubated in $40 \%$ follicular fluid for $120 \mathrm{~min}$.

* Statistically different from untreated follicular fluid values at least at $P=0.01\left(\chi^{2}\right.$ analysis $)$.

(Mukerjee \& Lippes, 1972). However, this activity is not confined to follicular fluid because the serum of many species effects these changes in hamster spermatozoa (Yanagimachi, 1970; Talbot, Franklin \& Fussell, 1974).

No significant activity was lost when the follicular fluids were dialysed (Table 1), indicating and confirming (Yanagimachi, 1969) the macromolecular nature of the effective component(s) (mol. wt $>10,000$ ). For the hamster, Yanagimachi (1969) noted the requirement for a small dialysable component for optimal results but such does not appear to be necessary for the rabbit.

Heating caused a 50-60\% loss of activity (Table 1). When bovine follicular fluid was heated for $0,5,15,30$ and $60 \mathrm{~min}$ and used to induce the acrosome reaction, $50 \%$ of the activity $(36 \%$ of spermatozoa reacted at $0 \mathrm{~min}$ ) was lost after $5 \mathrm{~min}(18 \%)$ and there was only $64 \%$ loss after $60 \mathrm{~min}(13 \%)$. Heating followed by dialysis produced results similar to those of heating alone. It seems possible that two forms of activity were present, one being due to a very labile material and the other being relatively heat stable.

Follicular fluids have been reported as having a toxic effect on spermatozoa (Yanagimachi, 1969; Mukerjee \& Lippes, 1972) and this has been overcome in some investigations by heating follicular fluid for $20-30 \mathrm{~min}$ at $56^{\circ} \mathrm{C}$. Except for the experiments described above, we did not heat the follicular fluid samples used. Approximately $30 \%$ of the samples caused immobilization of spermatozoa (often because the spermatozoa were trapped in the coagulated follicular fluid), while in the remaining samples spermatozoa were viable for the duration of the experiment and motility ranged from 50 to $75 \%$. Any samples in which $<50 \%$ of the spermatozoa were motile were excluded from the results.

\section{Concentration of follicular fuid and spermatozoa}

The extent of induction of the rabbit acrosome reaction was directly dependent on the bovine follicular fluid concentration (Table 2) as found by Yanagimachi (1969) for hamster spermatozoa. When the follicular fluid concentration of the assay mixture was maintained at $40 \%$ and the sperm concentration was varied, a dependence on sperm concentration was observed Text-fig. 1 . If the regression line in Text-fig. 1 is extrapolated to low sperm concentrations, as would be the case under

Table 2. Dependence of induction of the rabbit acrosome reaction ( $\%$ of completely reacted spermatozoa) on bovine follicular fluid concentration*

\begin{tabular}{ccccc}
\hline & \multicolumn{5}{c}{ Follicular fluid conc. (\%) } \\
\cline { 2 - 5 } Time & 0 & 20 & 40 & 80 \\
\hline $60 \mathrm{~min}$ & $2 \pm 1 \cdot 1$ & $10 \pm 2 \cdot 8$ & $28 \pm 5 \cdot 7$ & $41 \pm 11$ \\
$120 \mathrm{~min}$ & $2 \pm 2 \cdot 1$ & $21 \pm 3 \cdot 8$ & $42 \pm 4 \cdot 1$ & $67 \pm 1 \cdot 5$ \\
\hline
\end{tabular}

*The final incubation mixture had a sperm concentration of $1 \times 10^{7} / \mathrm{ml}$ and was incubated at $37^{\circ} \mathrm{C}$ under oil. Values are mean \pm S.D. for 6 determinations. 
physiological conditions, about $75 \%$ of the spermatozoa would undergo the acrosome reaction in $2 \mathrm{~h}$. This is in general agreement with the findings of Talbot et al. (1974) for the hamster acrosome reaction induced by sera at various sperm concentrations.

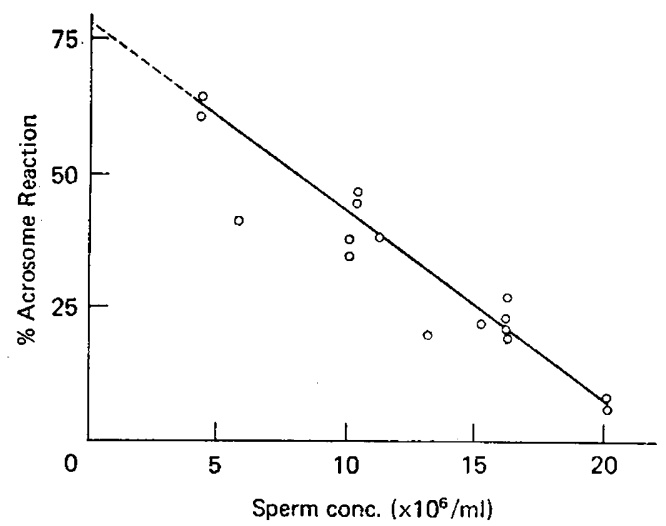

Text-fig. 1. Dependence of induction of the rabbit acrosome reaction on sperm concentrations. After sperm pretreatment incubations were in a final concentration of $40 \%$ bovine follicular fluid at $37^{\circ} \mathrm{C}$ under oil. Each point is the average of 2 determinations and represents spermatozoa from 5 different bucks.

$p H$

When the effect on the acrosome reaction of $\mathrm{pH}$ values $5,6,7,8,9$ and 10 was examined the response (mean \pm S.D., 3 determinations, $2 \mathrm{~h}$ incubation) was $0 \pm 2 \cdot 1,10 \cdot 5 \pm 1 \cdot 5,20 \pm 4 \cdot 1,29 \pm 3 \cdot 8$, $11 \pm 3 \cdot 2$ and $3 \pm 2 \cdot 3 \%$ reacted spermatozoa, respectively. The optimum response of $\mathrm{pH} 8$ would presumably be similar to that in the oviduct, the $\mathrm{pH}$ of which is about $7 \cdot 8$ (Hamner \& Williams, 1965). The activity of the acrosome reaction induced was irreversibly lost at $\mathrm{pH} 5$ but not totally destroyed at $\mathrm{pH} 10$. Activity in the control samples was $5-10 \%$ at $\mathrm{pH} 9$ and 10 and $<2 \%$ at $\mathrm{pH} 8$ or lower.

\section{Hydrolytic enzymes}

Treatment of follicular fluid with various hydrolytic enzymes (Table 3) indicated that proteases destroyed the activity of the acrosome reaction inducer but other enzymes had no significant effect. When spermatozoa were treated with the enzymes alone they were similar in appearance and motility to untreated controls. Deleterious effects resulted from pronase treatment, however: there was some separation of heads from tails and decreased motility, but the acrosomes still appeared intact.

To eliminate the possibility that the effect of the proteolytic enzymes was on the spermatozoa rather than on the follicular fluid, follicular fluid was pretreated with Agarose-bound trypsin. Most of the acrosome reaction-inducing activity was destroyed by the trypsin treatment: control untreated

Table 3. The effect of enzymatic treatment of bovine follicular fiuid on the rabbit acrosome reaction ( $\%$ complete)

\begin{tabular}{llc}
\hline \multicolumn{1}{c}{ Enzyme } & $\begin{array}{c}\text { Treatment } \\
\text { of sperm. }\end{array}$ & $\begin{array}{c}\text { Treatment of } \\
\text { follicular fluid }\end{array}$ \\
\hline$\alpha$-Amylase & $4 \pm 2.8$ & $35 \pm 1.6$ \\
$\beta$-Amylase & $3 \pm 1.6$ & $33 \pm 3.0$ \\
Lysozymes & $0 \pm 0$ & $30 \pm 3 \cdot 0$ \\
Lipase & $1 \pm 1$ & $30 \pm 1.0$ \\
Pronase & $0 \pm 0$ & $0 \pm 0$ \\
Trypsin & $0 \pm 0$ & $0 \pm 0$ \\
Control medium & $2 \pm 1.0$ & $35 \pm 7.5$ \\
\hline
\end{tabular}

Values are mean \pm S.D. of 3 experiments. 

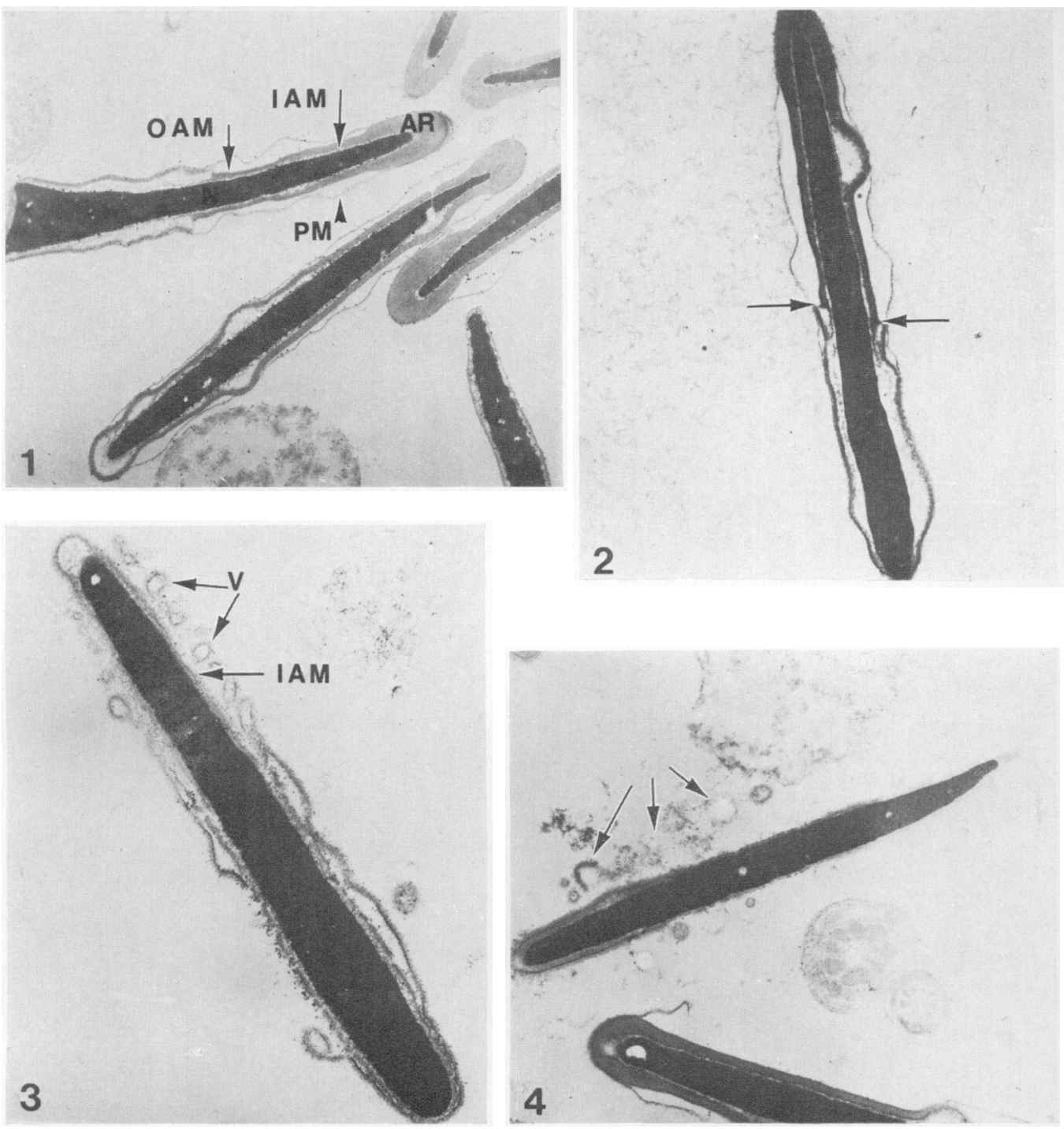

Fig. 1. Thin section of ejaculated rabbit spermatozoa after incubation for $30 \mathrm{~min}$ in CB medium (no HISmedium pretreatment). The nucleus $(\mathrm{N})$ is surrounded over the anterior aspect by the acrosome (AR) which is enclosed by the inner (IAM) and outer (OAM) acrosomal membranes. The plasma membrane (PM) is continuous over the head. $\times 9800$.

Fig. 2. Tangential section of a spermatozoa preincubated for $5 \mathrm{~min}$ in HIS medium followed by a 30-min incubation in $\mathrm{CB}$ medium. The acrosome and its surrounding membranes remain intact. Arrows indicate the equatorial segment. $\times 890$.

Fig. 3. Tangential section of a spermatozoon pretreated in HIS medium and incubated for $30 \mathrm{~min}$ in the presence of bovine follicular fluid. Fusion has occurred between the outer acrosomal and plasma membranes with loss of the acrosomal substance. The inner acrosomal membrane (IAM) remains intact. V, vesicles. $\times 17,100$.

Fig. 4. Terminal stage of the acrosome reaction. The acrosomal cap has been shed and the contents released. All that remains is the inner acrosomal membrane with some adherent electron-dense material. Arrows indicate remnants of acrosomal material. $\times 12,100$. 
follicular fluid gave $41 \pm 4.1 \%$ (S.D., 6 determinations) induction while trypsin-treated follicular fluid gave $0 \cdot 5 \pm 2.1 \%$ induction. However, the surface of the spermatozoa could still have been affected by the trypsin in the experiments in which direct exposure occurred, and it is possible that trypsin-like enzyme within the spermatozoa could play a role in the acrosome reaction as suggested by Meizel \& Lui (1976). The finding does, however, suggest that the acrosome reaction-inducing activity can be destroyed by proteolytic enzymes and that a protein is the effective follicular fluid component.

\section{Calcium ion}

Dialysis of follicular fluid against EDTA followed by dialysis against CB medium without calcium resulted in a decreased activity from $27 \pm 2.5 \%$ to $5 \pm 1 \%$ acrosome reactions, i.e. a loss of $>80 \%$ of the reaction-inducing activity. When the EDTA-dialysed follicular fluid was redialysed against normal CB medium (containing calcium), $25 \pm 2 \%$ acrosome reactions were induced in the same time, indicating that there was little or no loss of activity.

This calcium dependence is consistent with that reported by Yanagimachi \& Usui (1974) for induction of the hamster acrosome reaction. $\mathrm{Na}^{+}, \mathrm{K}^{+}$and $\mathrm{Mg}^{++}$are not involved because these are still present in the medium. In contrast to findings with the hamster, however, the rabbit acrosome reaction is not induced by calcium alone, as demonstrated by the negligible loss of acrosomes in control medium which contained $1.71 \mathrm{~mm}-\mathrm{CaCl}_{2}$.

\section{Antiserum}

When antiserum directed against bovine serum components was used to pretreat bovine follicular fluid, induction of the acrosome reaction was completely inhibited, the \% of acrosome-reacted spermatozoa being $2 \pm 1 \cdot 5$ (S.D., 6 determinations) compared with $28 \pm 3.8$ for follicular fluid (40\%) alone. The antiserum alone had little effect on the spermatozoa $(3 \pm 1.8 \%$ acrosome reaction) within the 120 -min incubation period. These results indicate that the factors in follicular fluid which induce the acrosome reaction are also present in serum. This is consistent with the results of others who showed that 'heat detoxified' serum will induce the hamster acrosome reaction (Yanagimachi, 1970; Talbot et al., 1974). Albumin has been implicated as the protein factor necessary in the hamster acrosome reaction (Yanagimachi, 1970), but this is not the sole factor required for the rabbit reaction. Our experiments do not exclude a role for albumin but concentrations of 4-30 $\mathrm{mg}$ albumin $/ \mathrm{ml}$ alone in a balanced salt medium do not induce the rabbit sperm acrosome reaction.

\section{Ultrastructural observations}

After incubation in control media (Pl. 1, Fig. 1), capacitation in HIS and incubation for $30 \mathrm{~min}$ in control medium (Pl. 1, Fig. 2), the sperm membranes were intact and ultrastructurally unchanged. After capacitation in HIS medium and incubation in follicular fluid (Pl. 1, Figs 3 and 4), the spermatozoa showed vesicle formation between the outer acrosome and the plasma membrane, the inner acrosomal membrane remaining intact, the apparent release of the contents of the acrosome and the ultimate loss of the outer acrosome membrane and adjacent plasma membrane.

These observations, like those relating to successful fertilization in vitro (Rosado et al., 1974; Oliphant, 1976), suggest that the changes in the rabbit acrosome induced by follicular fluid in vitro are similar to those of the acrosome reaction in vivo.

This work was supported by USPHS Grant R01-HD08573.

\section{References}

Barros, C., Bedford, J.M., Franklin, L.E. \& Austin, C.R. (1967) Membrane vesiculations as a feature of the mammalian acrosome reaction, J. Cell Biol. 34, C1-51.

Downloaded from Bioscientifica.com at 04/26/2023 06:20:37AM 
Brackett, B.G. \& Oliphant, G. (1975) Capacitation of rabbit spermatozoa in vitro. Biol. Reprod. 12, 260274.

Cross, P.C. \& BRINSTER, R.L. (1970) In vitro development of mouse oocytes. Biol. Reprod. 3, 298-307.

FAWCETT, D.W. (1958) Structure of the mammalian spermatozoon. Int. Rev. Cytol. 7, 195-263.

Hamner, C.E. \& Williams, W.L. (1965) Composition of rabbit oviduct secretions. Fert. Steril. 16, 170-176.

Iwamatsu, T. \& Chang, M.C. (1969) In vitro fertilization of mouse eggs in the presence of bovine follicular fluid. Nature Lond. 224, 919-920.

MEIZEL, S. \& LUI, C.W. (1976) Evidence for the role of a trypsin-like enzyme in the hamster sperm acrosome reaction. J. exp. Zool. 195, 137-144.

MUKERJEE, A.B. \& Lippes, J. (1972) Effect of human follicular and tubal fluids on human, mouse, and rat spermatozoa in vitro. Can. J. Genet. Cytol. 14, 167.

OLIPHANT, G. (1976) Removal of sperm bound seminal plasma components as a prerequisite to induction of the rabbit acrosome reaction. Fert. Steril. 27, 28-38.
Rosado, A., Hicks, J.J., Reyes, A. \& Blanco, 1. (1974) Capacitation in vitro of rabbit spermatozoa with cyclic-AMP and human follicular fluid. Fert. Steril. 25, 821-824.

Talbot, P., Franklin, L.E. \& Fussell, E.N. (1974) The effect of the concentration of golden hamster spermatozoa on the acrosome reaction and egg penetration in vitro. J. Reprod. Fert. 36, 429-432.

Wells, M.E. \& AWA, O.A. (1970) New techniques for assessing acrosomal characteristics of spermatozoa. J. Dairy Sci. 53, 227-232.

YANAGIMACHI, R. (1969) In vitro acrosome reaction and capacitation of golden hamster spermatozoa with bovine follicular fluid and its fractions. $J$. Reprod. Fert. 18, 275-286.

YANAGIMACHI, R. (1970) In vitro capacitation of golden hamster spermatozoa by homologous and heterologous blood sera. Biol. Reprod. 3, 147-153.

YANAGIMACHI, R. \& UsUI, N. (1974) Calcium dependence of the acrosome reaction and activation of guinea pig spermatozoa. Expl Cell Res. 89, $161-174$.

Received 30 September 1976 